\section{Atenção primária à saúde: estrutura das unidades como componente da atenção à saúde}

\section{Primary health care: the structure of units as a component of health care}

Bárbara Laisa Alves Moura 1

Renata Castro da Cunha 2

Ana Carla Freitas Fonseca 3

Rosana Aquino 4

Maria Guadalupe Medina 5

Ana Luiza Queiroz Vilasbôas 6

Aline Lima Xavier 7

Amanda Fortes Costa8

1-6 Programa Integrado de Pesquisa e Cooperação Técnica em Formação e Avaliação da Atenção Básica. Instituto de Saúde Coletiva. Universidade Federal da Bahia. Av. Basílio da Gama, s.n. Campos Universitário. Canela. Salvador, BA, Brasil. CEP: 40.110040. E-mail: blamoura@hotmail.com

7,8 Departamento de Atenção Básica. Secretaria de Saúde do Estado da Bahia. Salvador, BA, Brasil.

\section{Resumo}

Objetivos: avaliar a estrutura das unidades de

Objectives: to assess the structure of the primary health care units in two municipalities, comparing the Family Health Units(FHU) and the conventional units.

Methods: a quantitative cross-sectional study was carried out using structured interviews and observation of the work routine of the teams as data collection techniques. Three components were evaluated: the physical environment, material resources, and staff.

Results: the findings provided evidence of structural shortcomings in all three components, these being more serious in the Family Health Units. The shortcomings in terms of physical structure were more serious in municipality $B$, where the items under evaluation were fund in less than $70 \%$ of FHUs. In both municipalities, less than $50 \%$ of units had basic equipment. In municipality $A$, all the conventional units had doctors and nurses, while in municipality B, 27.3\% of FHUs had no doctor. Shortcomings were revealed that jeopardize biosafety and there was an absence of structures that favor the development of the kind of collective intervention that is necessary for changing the model of care by placing more emphasis on health surveillance.

Conclusions: although the chains of causation that link the structure of health services to their effects are complex and involve various factors, the study findings reiterate the need for more attention to be paid to structural components when evaluating health management.

Key words Program evaluation, Primary health care, Family health saúde da atenção primária de dois municípios, comparando as unidades de saúde da família (USF) e as unidades de saúde convencionais (USC).

Métodos: estudo transversal, quantitativo, utilizou entrevistas estruturadas e observação da rotina de trabalho das equipes como técnica de coleta de dados. Foram avaliados três componentes: ambiente físico, recurso material e pessoal.

Resultados: os achados evidenciaram deficiências estruturais nos três componentes, mais, onde os itens avaliados foram observados em menos de $70,0 \%$ das USF. Nos municipios, menos de $50 \%$ das unidades possuíam equipamentos básicos. No municipio A, todas ESF contavam com médicos e enfermeiros e, no municipio B, 27,3\% das ESF estavam sem médico. Ressaltam-se as deficiências que comprometem a biossegurança dos procedimentos e a ausência de estruturas que favorecem o desenvolvimento de intervenções coletivas, necessárias à mudança do modelo assistencial na perspectiva da vigilância da saúde.

Conclusões: embora as redes causais que ligam a estrutura dos serviços de saúde aos seus efeitos sejam complexas e envolvam diversos fatores, os achados do estudo reiteram a necessidade de valorização dos componentes estruturais na avaliação e gestão de saúde.

Palavras-chave Avaliação de programas $e$ projetos de saúde, Atenção primária à saúde, Saúde da família 


\section{Introdução}

A atenção primária à saúde (APS) é entendida como o primeiro nível do sistema de serviços de saúde, devendo funcionar como porta de entrada preferencial, com ações resolutivas sobre os problemas de saúde, articulando-se com os demais níveis de complexidade, formando uma rede integrada de serviços. ${ }^{1}$

Para organização deste nível de atenção, nos últimos anos, a Estratégia de Saúde da Família (ESF) tem sido adotada, no país, como política prioritária nas três esferas do Sistema Único de Saúde (SUS), com o propósito de priorizar a implementação de intervenções de promoção da saúde e prevenção de agravos e transformar as bases do modelo assistencial, definido como combinações tecnológicas estruturadas em função de problemas (danos e riscos) que compõem o perfil epidemiológico de uma dada população e que expressam necessidades sociais de saúde historicamente definidas. ${ }^{2}$ Embora responsável pela maior expansão da rede de serviços públicos de saúde registrada até a atualidade no Brasil, cobrindo mais de $50 \%$ da população, a almejada reversão do modelo assistencial está longe de se completar, coexistindo no país um modelo assistencial convencional, ainda fortemente hegemônico, e o modelo de vigilância à saúde. 3

Entre as diversas iniciativas de consolidação da Estratégia de Saúde da Família implementadas pelo Ministério da Saúde destacamos três pela abrangência e potencial de desenvolvimento de capacidades institucionais: o Projeto de Expansão e Consolidação da Estratégia Saúde da Família (PROESF), viabilizando investimentos financeiros voltados para a adequação da rede de serviços nos municípios brasileiros com população maior que cem mil habitantes ${ }^{4}$; a implementação do projeto de Avaliação para Melhoria da Qualidade da Estratégia de Saúde da Família (AMQ), estabelecendo diretrizes que orientam as práticas, organização e funcionamento dos serviços que direcionam a atuação das equipes de saúde da família 5 e o estudo de meta-avaliação comissionado pelo Ministério da Saúde para avaliação dos estudos de linha de base do Proesf. 6

No que se refere ao aspecto normativo, a política nacional de atenção básica preconizou a valorização dos aspectos estruturais das unidades de saúde, como itens necessários à realização das ações de atenção primária, sendo destacados: uma lista de ambientes que devem estar presentes em cada unidade de saúde; os equipamentos e materiais adequados para o conjunto de ações propostas, a composição da equipe multiprofissional e a garantia dos fluxos de referência e contrarreferência para os serviços especializados. ${ }^{7}$

Os debates e divergências quanto à natureza e pertinência de orientações normativas, mesmo que flexíveis, para organização dos aspectos estruturais relacionados às unidades prestadoras de serviços e dos processos de cuidados, possivelmente, determinaram que a atualização destas normas, no âmbito da atenção primária, fosse relativamente recente. Entre os documentos oficiais que determinam critérios de estrutura para os estabelecimentos de saúde destacam-se a Resolução da Diretoria Colegiada (RDC) $\mathrm{n}^{\mathrm{o}} 50^{8}$ e o manual de estrutura física das unidades primárias de saúde, ${ }^{9}$ que apresentam a regulamentação técnica para planejamento, programação, elaboração e avaliação de projetos físicos de estabelecimentos assistenciais de saúde. Além destes, a Portaria GM 648/2006 estabelece a revisão de diretrizes e normas para a ESF (incluindo o Programa de Agentes Comunitários de Saúde, PACS) e especifica padrões de infraestrutura, recursos humanos e materiais necessários para o desenvolvimento das ações das equipes de saúde da família. 7

No campo da avaliação de saúde, a estrutura é um dos componentes destacados por Donabedian. 10 Sua abordagem se baseia no modelo sistêmico onde a estrutura corresponde àquilo que é relativamente estável no sistema (os recursos ou insumos financeiros, humanos e materiais utilizados), o processo, ao conjunto de atividades e procedimentos empregados no manejo dos recursos, e os resultados às mudanças verificadas, sejam elas relacionadas a um efeito no estado de saúde dos indivíduos, ou a mudanças de comportamentos, conhecimentos ou satisfação dos usuários dos serviços. ${ }^{11}$

Starfield ${ }^{1}$ também valoriza a estrutura como um dos componentes para análise do sistema de serviços de saúde, salientando a influência do comportamento individual e pelo ambiente físico, político, econômico e social, sobre este e os demais componentes. A estrutura, denominada pela autora como capacidade, corresponde àquilo que propicia a prestação dos serviços, ou seja, os recursos necessários para oferecer os serviços. Entre esses estão a análise de pessoal, que considera a educação e treinamento de todos os envolvidos na prestação do serviço e a análise da instalação e equipamentos, que inclui o prédio e os componentes físicos da instalação.

Segundo Vuori,12 a maior fraqueza da abordagem estrutural está relacionada com seus pressupostos de validade, ou seja, a presunção que uma boa 
estrutura leva a um bom processo e este, por sua vez, a um bom resultado. Como pondera o autor, no caso da atenção à saúde, as cadeias que ligam a estrutura aos resultados são longas e, geralmente, apresentam ligações fracas, insustentáveis logicamente e não comprovadas. Assim, as melhores pré-condições podem ser mal utilizadas, enquanto a excelência profissional pode resultar em efeitos benéficos, mesmo em condições adversas.

Como a abordagem estrutural, a abordagem de processo tem sido muito criticada pela impossibilidade do estabelecimento de relações causais entre os procedimentos realizados e a melhoria das condições de saúde das pessoas. A avaliação de resultados seria, então, aquela de maior validade para medir a qualidade da atenção prestada. Entretanto, em que pese a aparente primazia da abordagem de resultados, há que considerar que a melhoria da atenção à saúde ocorre ao nível do processo ou da estrutura, pois os resultados são sempre consequência de alguma coisa e para seu alcance há que identificar as ações corretivas necessárias nos serviços e nas práticas sanitárias. ${ }^{12,13}$

Concordando que na prática concreta dos serviços de saúde não existe ordem, harmonia nem a direcionalidade evocadas por modelos sistêmicos, 13 o presente estudo tem como objetivo avaliar a estrutura das unidades de saúde da atenção primária de dois municípios que aderiram à ESF, buscando destacar um tema negligenciado na organização da atenção primária, ou seja, os efeitos nefastos da precariedade da estrutura dos serviços sobre a qualidade dos processos de trabalho em saúde e organização dos modelos de atenção à saúde.

\section{Métodos}

Este estudo faz parte da pesquisa intitulada "Caracterização da atenção primária em municípios que aderiram ao programa saúde da família no Estado da Bahia", que teve como objetivo caracterizar a modalidade de organização da atenção primária em dois municípios que aderiram à ESF, aqui denominados município A e município B. Os municípios foram selecionados considerando os seguintes critérios: população maior de $100 \mathrm{mil} \mathrm{habi-}$ tantes e ESF implantada há pelo menos dois anos. ${ }^{14}$

O município A possuía, em 2007, uma população de, aproximadamente, 220.000 habitantes, sendo que a história da organização municipal caracterizava-se por um crescimento acelerado, sustentado amplamente pelo desenvolvimento industrial da região. Destaca-se a grande extensão territorial do município, com grandes diferenças quanto à distribuição populacional, especialmente no que se refere às características socioeconômicas. O perfil sanitário do município retrata a transição epidemiológica do país, em que a população convive com os antigos e modernos fatores de riscos, com sobreposição de uma carga, ainda presente, mas cada vez menor, de doenças infecciosas e parasitárias que afetam principalmente as crianças, com o predomínio das doenças crônico-degenerativas e dos agravos relacionados à violência.

O município $\mathrm{B}$, com população em torno de 132.000 habitantes, no mesmo período, apresentava como principal fonte de renda o comércio, acrescida de indústrias de pequeno porte. A organização do espaço urbano no município se deu de forma desordenada e tal característica trouxe consequências para a população local, principalmente no que se refere à infraestrutura, como problemas de pavimentação urbana e acesso a saneamento básico. Seu perfil epidemiológico era semelhante ao perfil do município A.

Quanto à organização do sistema de saúde, ambos apresentavam-se habilitados na gestão plena do sistema municipal de saúde, segundo a norma operacional básica (NOB 96, no caso do município A) e norma operacional de assistência à saúde (NOAS 2001, município B). A administração dos serviços da atenção primária está a cargo da gestão pública. Entretanto, identifica-se nos dois municípios uma importante participação do setor privado na oferta de procedimentos de média e alta complexidade no âmbito dos serviços contratados pelo SUS.

O estudo foi do tipo transversal e utilizou uma abordagem quantitativa. A unidade de coleta e análise dos dados foi a unidade de saúde de atenção primária. Em um dos municípios onde duas unidades funcionavam com duas equipes de saúde da família, os dados foram analisados por equipe, sendo estas tratadas como unidades de saúde.

Foram realizadas entrevistas semiestruturadas com os coordenadores, gerentes das equipes de saúde ou profissionais com função semelhante, além de observação, guiada por roteiro, da rotina de trabalho das equipes e estrutura organizacional. Foram avaliadas todas as unidades da atenção primária à saúde, sendo 24 unidades de saúde da família (USF) e 9 unidades de saúde convencionais (USC) no município A e 22 USF e 1 USC no município B. Os dados de saúde bucal correspondem ao total de equipes de saúde bucal (ESB) existentes nas unidades avaliadas, sendo 9 em USF e $9 \mathrm{em}$ USC, no município A e 15 em USF e uma na USC no município $\mathrm{B}$.

A coleta de dados foi realizada no período de 
maio a junho de 2007 por profissionais de saúde de nível superior previamente treinados e com supervisão de campo. A coleta foi precedida de um estudo piloto realizado no município de Salvador, sendo o instrumento aplicado em USC e USF.

A estrutura foi analisada a partir dos seguintes componentes: ambiente físico, recursos materiais e pessoal. Para a construção das variáveis foram adaptadas classificações utilizadas por documentos do Ministério da Saúde. 9

$\mathrm{O}$ ambiente físico foi avaliado considerando a existência, compartilhamento dos espaços, condições de conservação (ventilação, iluminação e umidade) e higiene das dependências. As variáveis para caracterização do ambiente físico foram: dependências básicas (existência de sala de recepção ou espera, consultório médico e de enfermagem, sala de procedimentos, sala de imunização, sala de curativo, expurgo e dois sanitários); consultório odontológico; copa; Serviço de Arquivos Médicos e de Estatística (SAME); almoxarifado, esterilização; sala de reunião; abrigo de resíduos sólidos e farmácia.

Foram considerados como recursos materiais os equipamentos e os insumos necessários ao desenvolvimento das ações de atenção primária. Todos os recursos foram classificados quanto à existência e suficiência para uso segundo a percepção dos entrevistados. As variáveis utilizadas foram: equipamentos básicos (estetoscópio, tensiômetro adulto, fita métrica, termômetro, maca, balança antropométrica e balança infantil); equipamento ginecológico (foco, mesa ginecologia, espéculos ginecológicos), sonar; equipamento audiovisual (aparelho de vídeo e/ou aparelho de DVD e televisão), equipamento odontológico básico (autoclave, cadeira e equipo com pontas, amalgamador, refletor, mocho, cuspideira), biombo duplo, tensiômetro infantil, otoscópio, lanterna, nebulizador, mesa auxiliar, antropômetro, geladeira exclusiva para vacina, glicosímetro com fitas, oftalmoscópio, material de emergência (equipamento e medicação), fotopolimerizador, aparelho de profilaxia, negatoscópio. Quanto aos insumos, as variáveis foram: medicamentos, formulários e fichas de atendimento, equipamento de proteção individual (EPI) e materiais usados para curativo, retirada de pontos, atendimento odontológico, educação em saúde, pequena cirurgia e limpeza.

$O$ pessoal foi analisado por categoria profissional de nível superior (médico, odontólogo e enfermeiro), considerando o quantitativo, o tempo de inserção na equipe e a carga horária.

Foram criadas categorias para codificação dos dados obtidos pelas entrevistas e roteiro de observação. Os dados obtidos através das entrevistas foram cotejados com os dados obtidos mediante observação dos entrevistadores. Foi realizada análise tabular, sendo calculadas as frequências para cada variável. Alguns aspectos foram comparados por tipo de unidade (USF e USC) e área de atuação (rural e urbana). Os dados quantitativos foram processados e analisados com a utilização dos softwares Excel e Stata(versão 9.0). Foi obtida a anuência dos gestores municipais de saúde e da gerência das unidades para a coleta dos dados. As entrevistas foram realizadas após consentimento informado dos sujeitos, conforme termo específico, que assegurava o anonimato. Este estudo foi aprovado pelo Comitê de Ética do Instituto de Saúde Coletiva da Universidade Federal da Bahia (Registro 060-06/CEP-ISC).

\section{Resultados}

O município A possuía 33 unidades, sendo 12 unidades de saúde localizadas na Zona Rural (ZR) e 21 unidades de saúde localizadas na Zona Urbana (ZU), com 24 ESF cobrindo 43,1\% da população e 9 USC. A mediana de tempo de funcionamento das USC foi de 20 anos e das USF, de 7 anos. Destaca-se que a primeira equipe USF foi implantada em 1998, com rápido crescimento nos dois anos subsequentes. A implantação de equipes de saúde bucal (ESB) vinculadas à ESF ocorreu em 2004.

O município B possuía 23 unidades, uma USC em área urbana e 22 USF, totalizando 6 unidades de saúde na ZR e 17 unidades na ZU. No início da implantação da ESF em 1998, observou-se a substituição das unidades de saúde convencionais pelas unidades de saúde da família. Em 2001, o programa apresentou importante expansão, quando foram implantadas 14 equipes, e em 2006, a ESF alcançava $57,4 \%$ de cobertura populacional, mantendo-se em 2007. A mediana de tempo de funcionamento das USF foi de seis anos.

Os dois municípios apresentavam deficiências quanto à estrutura física, mais acentuadas no município $\mathrm{B}$, onde todos os itens avaliados foram observados em menos de $70,0 \%$ das unidades de saúde da família. No município A evidenciou-se que apenas $12,5 \%$ das USF e $55,6 \%$ das USC apresentavam as dependências básicas. Estes percentuais elevam-se para $25,0 \%$ nas USF quando não se considera o expurgo. Menos de $25,0 \%$ das unidades apresentavam sala de reunião $(20,8 \%$ nas USF e $11,1 \%$ nas USC) e, no caso das USF, apenas $12,5 \%$ apresentavam abrigo de resíduos sólidos. No município $\mathrm{B}$, apenas $4,5 \%$ das USF apresentavam as 
dependências básicas, percentual que se eleva para $9,0 \%$ quando não se considera o expurgo. Menos de $25,0 \%$ das unidades apresentavam sala de reunião (13,6\% nas USF e $0 \%$ na USC), abrigo de resíduos sólidos (13,6\% na USF e $0 \%$ na USC) e SAME $(22,7 \%$ nas USF). Nos dois municípios não foram observadas diferenças expressivas entre as zonas urbanas e rurais, a exceção da presença de farmácia nas unidades do município $B$ que foi maior na Zona Urbana (ZU) (64,7\%) do que na Zona Rural (ZR) $(16,7 \%)$, como se pode observar na Tabela 1 .

Observou-se a existência de condições inadequadas de biossegurança, uma vez que procedimentos que devem ser realizados em condições assépticas e aqueles que interferem na manutenção dessas eram efetuados no mesmo local. Verificou-se precário estado de conservação e higiene das unidades, com presença de infiltrações e iluminação deficiente, além da ausência de rampa de acesso e sanitário adaptado para atender aos pacientes portadores de necessidades especiais. Destaca-se que muitas USF, especialmente no município B, encontravam-se sediadas em estruturas físicas que não foram construídas para tal fim, como casas adaptadas, que não atendiam às normas preconizadas para o funcionamento de serviços de saúde.

Em relação aos recursos materiais utilizados para assistência ao paciente, no município A, 75,0\% ou mais das unidades apresentavam suficiência de material de curativo e de retirada de pontos, mais de $65,0 \%$ para material odontológico e menos de $35,0 \%$ quanto aos medicamentos e material para pequena cirurgia. À exceção de material odontológico, os percentuais foram mais baixos para as unidades de saúde da família. No município $\mathrm{B}$, nas unidades de saúde da família, cerca de $40,0 \%$ apresentavam suficiência de material de curativo, $45,5 \%$ de medicamentos, menos de $65,0 \%$ para material de retirada de pontos, $80,0 \%$ para material odontológico e menos de $10,0 \%$ para material para pequena cirurgia. No caso da USC, medicamentos e material para curativo foram considerados insuficientes. Na maioria das unidades de saúde dos dois municípios foi referido que não havia suficiência de material educativo para desenvolvimento das atividades (Tabela 2).

Destaca-se que em apenas $41,7 \%$ das USF do município A foi referida suficiência de material de limpeza, situação observada em $88,9 \%$ das USC deste município e em mais de $80,0 \%$ das unidades do município B. Suficiência de formulários e fichas foi referida em pouco mais da metade das unidades do município A e em menos de 30,0\% do município B. Em relação à EPI, esta situação foi observada em $88,9 \%$ ou mais das unidades (Tabela 2 ).
Para a maioria dos itens investigados, a situação das unidades da zona rural foi pior do que as da zona urbana no município A, ressaltando a insuficiência de material de limpeza e odontológico, enquanto no município B observou-se o inverso, especialmente quanto aos medicamentos e material odontológico (Tabela 2).

O percentual de unidades que possuíam os equipamentos básicos, no município $\mathrm{A}$, era inferior a $35 \%$, maior na ZR do que na ZU. No município B, $45,5 \%$ das USF possuíam os equipamentos básicos, com pouca diferença entre as ZU e ZR. A USC do município não apresentava o conjunto de equipamentos considerados básicos.

Em relação aos demais equipamentos, menos de $50 \%$ das unidades de saúde do município A possuíam tensiômetro infantil, otoscópio, lanterna, oftalmoscópio, material de emergência, equipamentos odontológicos e audiovisuais e negatoscópio no caso das USF. No município B, além dos citados, menos da metade das USF apresentava glicosímetro com fitas e aparelho de profilaxia odontológica. A USC do município B não dispunha de lanterna, antropômetro, negatoscópio, material de emergência, aparelho de profilaxia e biombo duplo. Nenhuma das unidades deste município possuía oftalmoscópios e equipamentos audiovisuais (Tabela 3 ).

A rede de atenção primária no município $\mathrm{A}$ contava com 101 médicos, 30 odontólogos e 62 enfermeiros, o que correspondia a uma relação de profissionais por 10.000 habitantes de 4,59, 1,36 e 2,81 , respectivamente. O município $\mathrm{B}$ apresentava 22 médicos, 17 odontólogos e 25 enfermeiros, sendo a proporção de profissionais por habitante bem mais reduzida, especialmente no que se refere aos médicos $(1,65)$. Para os demais profissionais, esta proporção equivalia a 1,28 para odontólogos e 1,89 para enfermeiros. Em ambos os municípios, a relação médico/paciente é inferior ao preconizado pelos documentos oficiais. 15

Ressalta-se que as informações sobre o tempo de inserção dos profissionais não foram obtidas para a totalidade de profissionais da rede, correspondendo a $78 \%$ dos médicos, $93 \%$ dos odontólogos e $88 \%$ dos enfermeiros. No município $A$, todas as ESF contavam com profissionais médicos e enfermeiros, o que não ocorreu no município $\mathrm{B}$, onde $27,3 \%$ das equipes estavam sem médicos no momento da avaliação. Equipes de saúde bucal foram encontradas em $45,4 \%$ das USF do município A e $68,2 \%$ das USF do município $\mathrm{B}$; entretanto uma equipe de cada município encontrava-se sem odontólogo.

Nos dois municípios o tempo de inserção dos profissionais nas unidades de saúde variou por tipo 


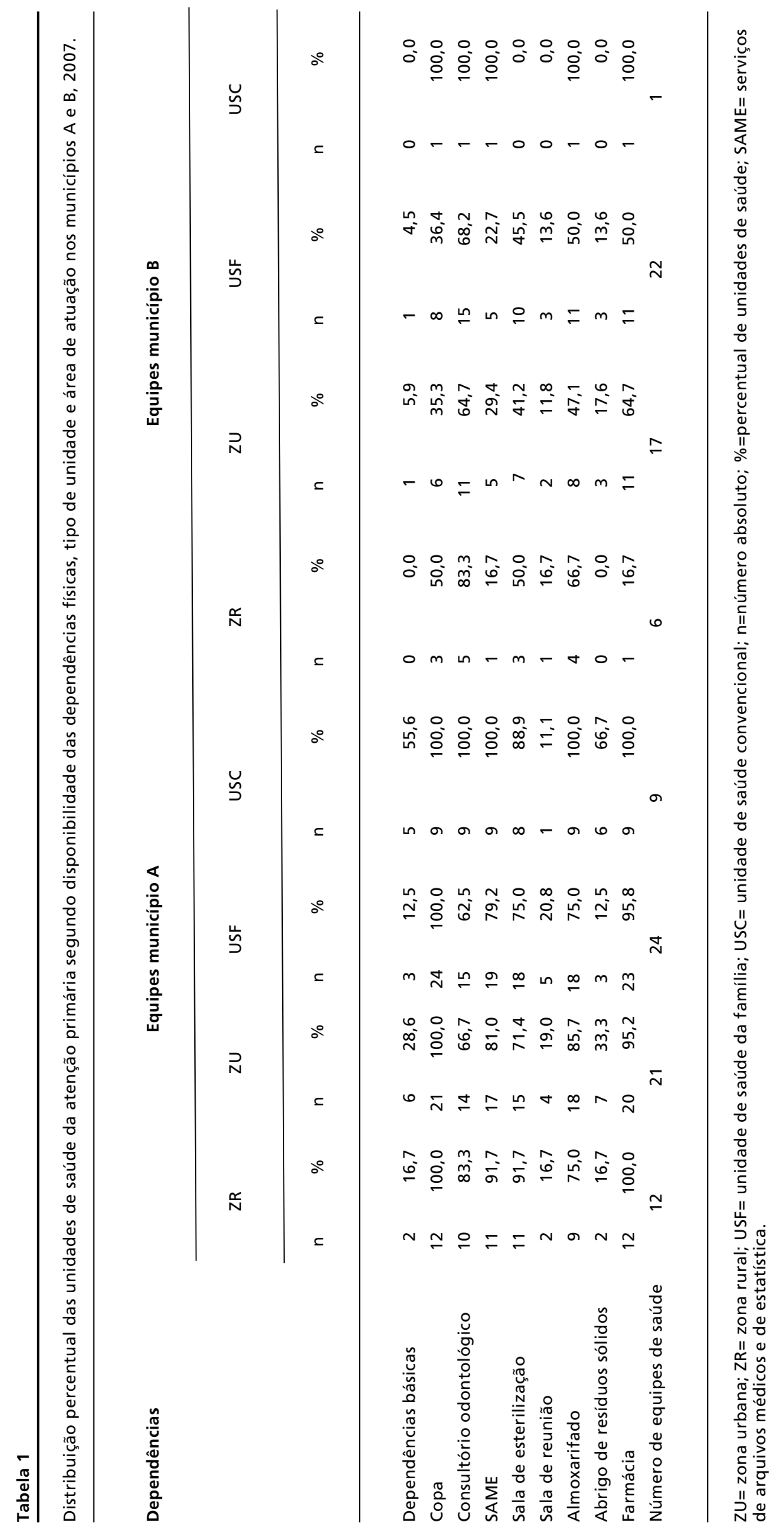




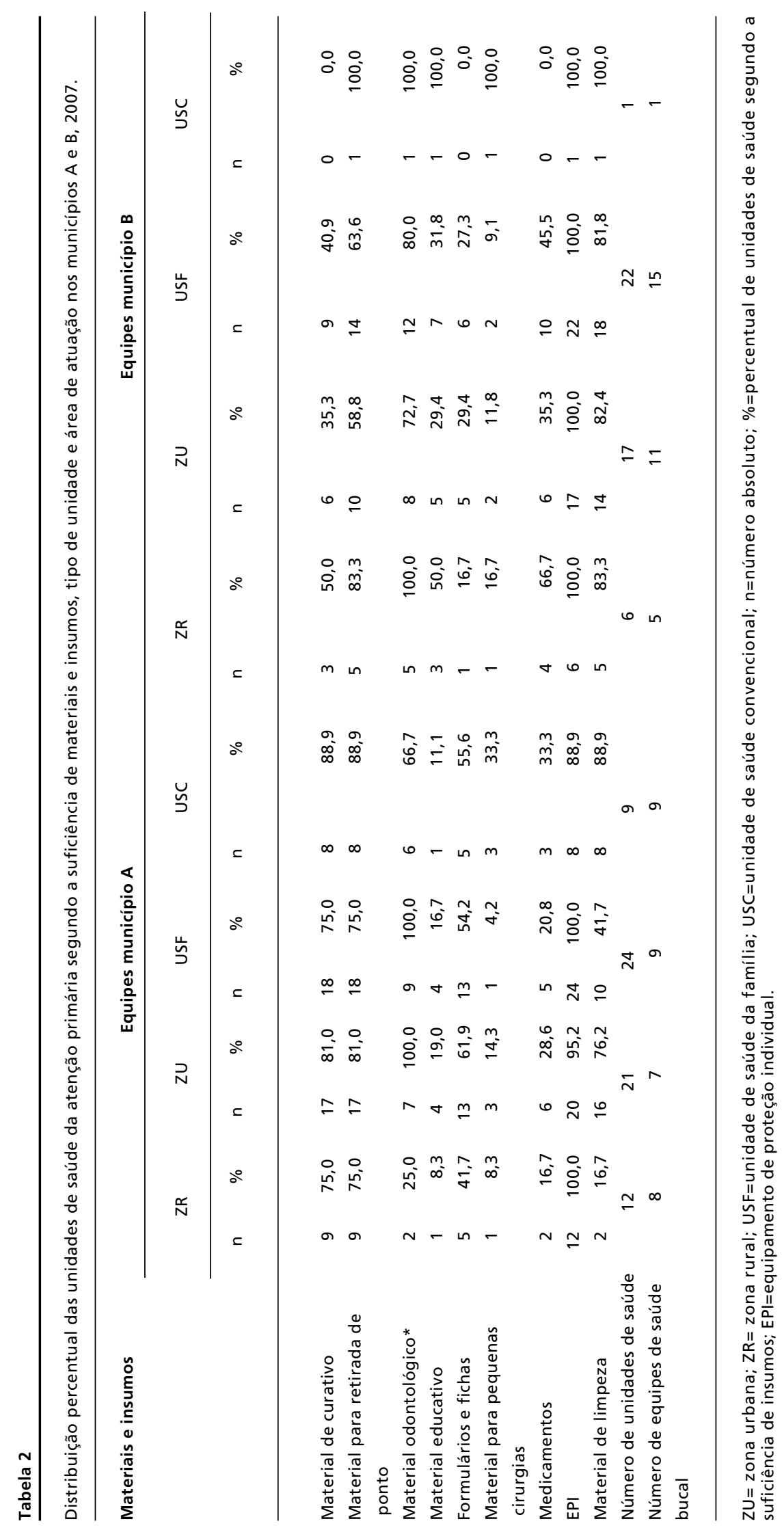




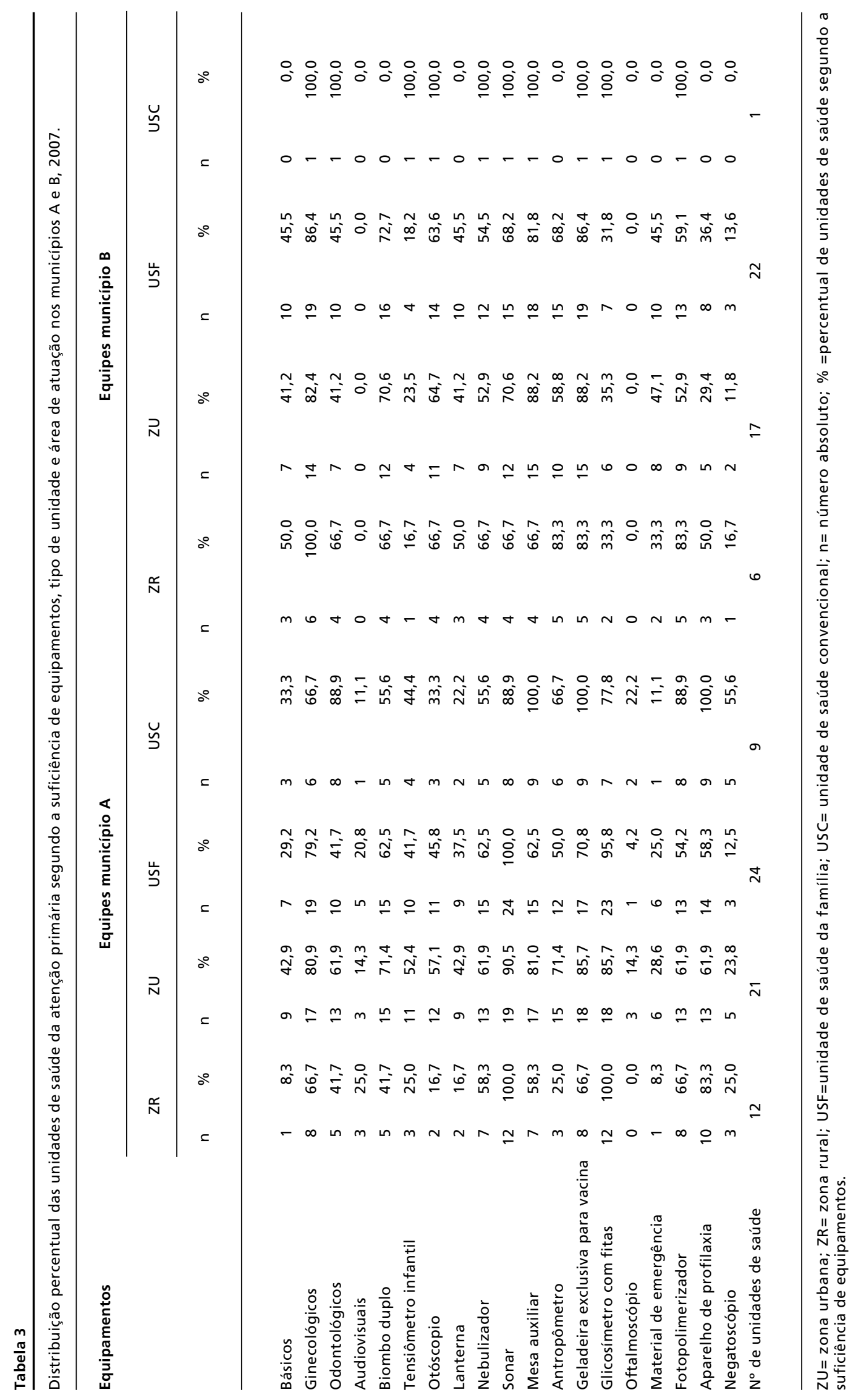


de categoria profissional e tipo de unidade. No município A, a maioria dos médicos e odontólogos apresentavam menos de dois anos de inserção, enquanto a maioria dos enfermeiros estavam inseridos há mais de dois anos nas USF. Nas USC, tal situação ocorreu de forma inversa. No município $\mathrm{B}$, a maioria das categorias profissionais analisadas apresentavam menos de dois anos de inserção, à exceção dos odontólogos das USF. O percentual de profissionais com mais de dois anos de inserção no município A foi maior na zona urbana no caso dos médicos e odontólogos, enquanto no município B foi maior na zona rural para enfermeiros e odontólogos (Tabela 4).
Quanto à carga horária dos profissionais das USF, no município A foi referido que a totalidade dos profissionais cumpria a carga horária recomendada e no município B este percentual foi de 50,0\% para médicos, $72,7 \%$ para enfermeiros e $53,8 \%$ para odontólogos. Ressalta-se que muitos profissionais do município B referiram dispor de um dia de folga por semana. Nas USC, foi referido nos municípios A e $\mathrm{B}$, respectivamente, que, $85,7 \%$ e $66,7 \%$ dos médicos, $75,0 \%$ e $100 \%$ dos odontólogos cumpriam até 20 horas semanais e $65,8 \%$ e $100 \%$ dos enfermeiros cumpriam 40 horas semanais (Tabela 5).

Tabela 4

Distribuição numérica e percentual dos profissionais segundo tempo de inserção, tipo de unidade e área de atuação, municípios A e B 2007.

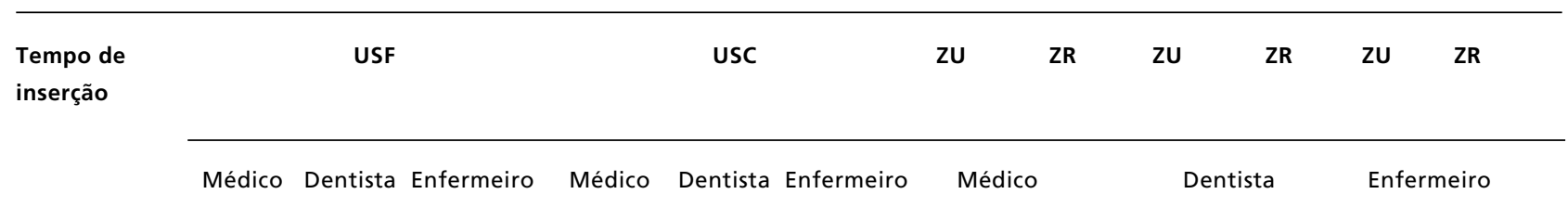

Município A

$<1$ ano

$\mathrm{n}$

$\%$

1 a 2 anos

n

$\%$

$>2$ anos

$\mathrm{n}$

$\%$

Total

Município B

$<1$ ano

$\mathrm{n}$

$\%$

1 a 2 anos

$\mathrm{N}$

$\%$

$>2$ anos

$\mathrm{n}$

$\%$

Total

$\begin{array}{ccc}12 & 5 & 10 \\ 50,0 & 35,7 & 41,7 \\ & & \\ 5 & 3 & 1 \\ 20,8 & 21,4 & 4,2 \\ & & \\ 7 & 6 & 13 \\ 29,2 & 42,9 & 54,2 \\ 24 & 14 & 24\end{array}$

$\begin{array}{lcc}11 & 1 & 13 \\ 20,0 & 7,1 & 41,9 \\ 13 & 3 & 11 \\ 23,6 & 21,4 & 35,5 \\ 31 & 10 & 7 \\ 56,4 & 71,4 & 22,6 \\ 55 & 14 & 31\end{array}$

$\begin{array}{lccccc}17 & 6 & 3 & 3 & 14 & 9 \\ 29,8 & 27,3 & 15,8 & 33,3 & 38,0 & 50,0 \\ 8 & 10 & 3 & 3 & 10 & 2 \\ 14,0 & 45,5 & 15,8 & 33,3 & 27,0 & 11,1 \\ 32 & 6 & 13 & 3 & 13 & 7 \\ 56,1 & 27,3 & 68,4 & 33,3 & 35,0 & 38,9 \\ 57 & 22 & 19 & 9 & 37 & 18\end{array}$

$Z U=$ zona urbana; $Z R=$ zona rural; USF=unidade de saúde da família; USC= unidade de saúde convencional; $n=$ número absoluto de profissionais por categoria; $\%=$ percentual de profissionais por categoria. 
Tabela 5

Distribuição numérica e percentual dos profissionais segundo carga horária, tipo de unidade e área de atuação, municípios A e B, 2007.

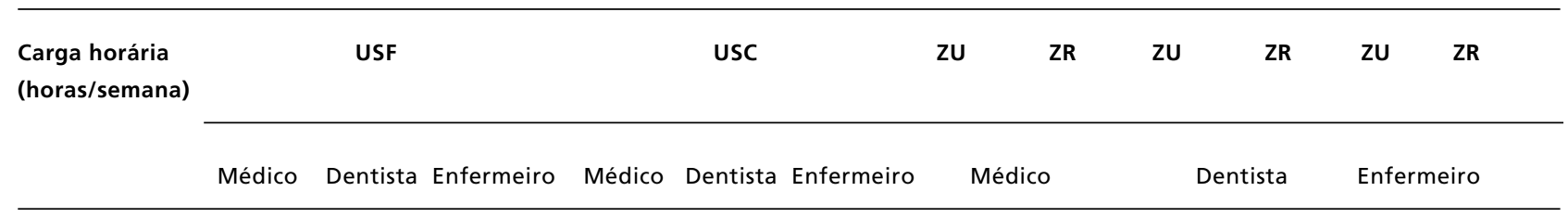

\begin{tabular}{|c|c|c|c|c|c|c|c|c|c|c|c|c|}
\hline \multicolumn{13}{|c|}{$\begin{array}{l}\text { Município A } \\
\leq 20\end{array}$} \\
\hline $\mathrm{n}$ & 0 & 1 & 0 & 66 & 12 & 2 & 56 & 10 & 10 & 3 & 2 & 0 \\
\hline$\%$ & 0,0 & 7,1 & 0,0 & 85,7 & 75,0 & 5,3 & 72,7 & 41,7 & 52,6 & 27,3 & 4,4 & 0,0 \\
\hline \multicolumn{13}{|l|}{$20-40$} \\
\hline $\mathrm{n}$ & 0 & 0 & 0 & 5 & 2 & 11 & 1 & 4 & 1 & 1 & 9 & 2 \\
\hline$\%$ & 0,0 & 0,0 & 0,0 & 6,5 & 12,5 & 28,9 & 1,3 & 16,7 & 5,3 & 9,1 & 20,0 & 11,8 \\
\hline \multicolumn{13}{|l|}{40} \\
\hline $\mathrm{n}$ & 24 & 13 & 24 & 6 & 2 & 25 & 20 & 10 & 8 & 7 & 34 & 15 \\
\hline$\%$ & 100,0 & 92,9 & 100,0 & 7,8 & 12,5 & 65,8 & 26,0 & 41,7 & 42,1 & 63,6 & 76,0 & 88,2 \\
\hline Total & 24 & 14 & 24 & 77 & 16 & 38 & 77 & 24 & 19 & 11 & 45 & 17 \\
\hline \multicolumn{13}{|c|}{ Município B } \\
\hline \multicolumn{13}{|c|}{$\leq 20$} \\
\hline $\mathrm{n}$ & 4 & 1 & 0 & 4 & 4 & 0 & 7 & 1 & 5 & 0 & 0 & 0 \\
\hline$\%$ & 25,0 & 7,7 & 0,0 & 66,7 & 100,0 & 0,0 & 41,2 & 20,0 & 38,5 & 0,0 & 0,0 & 0,0 \\
\hline \multicolumn{13}{|l|}{$20-40$} \\
\hline $\mathrm{n}$ & 4 & 5 & 6 & 1 & 0 & 0 & 4 & 1 & 4 & 1 & 5 & 1 \\
\hline$\%$ & 25,0 & 38,5 & 27,3 & 16,7 & 0,0 & 0,0 & 23,5 & 20,0 & 30,8 & 25,0 & 26,0 & 16,7 \\
\hline \multicolumn{13}{|l|}{40} \\
\hline $\mathrm{n}$ & 8 & 7 & 16 & 1 & 0 & 3 & 6 & 3 & 4 & 3 & 14 & 5 \\
\hline$\%$ & 50,0 & 53,8 & 72,7 & 16,7 & 0,0 & 100,0 & 35,3 & 60,0 & 30,8 & 75,0 & 74,0 & 83,3 \\
\hline Total & 16 & 13 & 22 & 6 & 4 & 3 & 17 & 5 & 13 & 4 & 19 & 6 \\
\hline
\end{tabular}

$Z U=$ zona urbana; $Z R=$ zona rural; USF=unidade de saúde da família; USC= unidade de saúde convencional; $n=$ número absoluto de profissionais por categoria; \%=percentual de profissionais por categoria.

\section{Discussão}

O estudo evidenciou deficiências nos três componentes de estrutura analisados (ambiente físico, recursos materiais e pessoal) em ambos os municípios, nas duas modalidades de unidades de saúde da atenção primária. As deficiências identificadas foram mais acentuadas no município $\mathrm{B}$, especialmente no componente ambiente físico e, ao comparar o tipo de unidade no município A, foram observadas maiores limitações nas USF.

O fato de poucos estudos abordarem a avaliação da estrutura, considerando os componentes estrutura física, equipamentos e recursos humanos, dificultou a comparação dos achados. É válido ressaltar que a classificação de suficiência dos insumos provém da percepção dos profissionais entrevistados, inexistindo uma classificação prévia do que seria considerado suficiente. A existência de apenas uma USC no município B limitou as comparações para esta categoria.

A avaliação do componente ambiente físico, em ambos os municípios, revelou a pouca observação dos princípios da Resolução da Diretoria Colegiada - RDC n ${ }^{\circ}$ 50/ANVISA (2002). ${ }^{8}$ Tais limitações interferem na manutenção da biossegurança, uma vez que grande parte das unidades de saúde não dispõe de sala específica para procedimentos, identificando-se a aplicação de medicamentos injetáveis no mesmo espaço no qual é realizado os curativos, além de pouca valorização do expurgo, sendo a descontaminação de instrumentais realizada em local inapropriado, conforme as especificações de biossegurança. 16

Resultados semelhantes foram encontrados no estudo de Rocha e Araújo, 17 o qual evidenciou a 
ausência de expurgo, sala de reuniões e outras dependências como elementos que dificultam o desenvolvimento das atividades do dentista na ESF. Neste estudo a estrutura física foi identificada como o fator que mais influenciou nas condições de trabalho dos odontólogos.

A ausência de sala de reunião em grande parte das unidades pode dificultar a realização de atividades coletivas com a comunidade e a articulação das práticas de avaliação e planejamento entre os profissionais da unidade. Tais ações são características da mudança de modelo de atenção, numa perspectiva da vigilância em saúde e, conforme aponta o Ministério da Saúde, ${ }^{9}$ a estrutura física das unidades de saúde deve ser facilitadora da mudança das práticas em saúde.

A limitação do acesso a idosos e portadores de deficiências também foi um aspecto evidenciado, ao verificar-se a ausência de rampa de acesso e sanitário adaptado na grande maioria das unidades nos dois municípios. Esse achado também se evidenciou no estudo de Siqueira et al.,18 que, ao avaliar a estrutura arquitetônica de uma amostra de unidades básicas de saúde de municípios em sete Estados brasileiros, classificou $60 \%$ das USC como inadequadas para o acesso de idosos e portadores de deficiências, com maior expressão nas USF quando comparadas às USC.

A frequente utilização de imóveis com características domiciliares para a instalação de USF, no município B, sem a adequada adaptação, é um aspecto que reforça a pouca valorização dos aspectos estruturais nas unidades de atenção primária, em particular nas USF, tal qual se encontrou em outros estudos.17-19 Tais deficiências podem ser reflexo da concepção de modelo de saúde da família adotado pelos municípios estudados, em que predominam unidades de saúde pequenas com apenas uma ESF. Conforme refletem Ximenes e Sampaio:20

\footnotetext{
[...] o que se espera da estrutura de uma unidade da ESF não é que seja perfeita ou padrão, mas que possa conformar no espaço todas as suas necessidades organizativas, assistenciais e da comunidade.
}

A insuficiência de recursos materiais também foi um aspecto destacado. A falta de equipamentos adequados, como geladeira exclusiva para vacina $\mathrm{e}$ autoclave, interfere na manutenção da biossegurança; assim como a insuficiência de material de limpeza, observada na maioria das USF do município A.

Destaca-se ainda a insuficiência de materiais educativos, formulários e fichas, medicamentos e materiais para pequenas cirurgias como fatores que influenciam sobre a qualidade da atenção aos usuários, uma vez que podem dificultar a realização de atividades de promoção à saúde, prevenção de agravos e comprometer a continuidade do cuidado. O número reduzido de USF com disponibilidade de material para pequenas cirurgias condiz com os resultados da pesquisa de Avaliação Normativa do Programa de Saúde da Família, realizada a nível nacional, onde apenas nove Estados brasileiros expressaram a disponibilidade deste material em mais de $50 \%$ das USF. 21

É válido ressaltar que era esperado que a disponibilidade de material educativo fosse referida por um número maior de USF, uma vez que a estratégia de saúde da família tem como uma de suas principais linhas de ação a promoção da saúde e prevenção de agravos. ${ }^{7}$

Neste estudo evidenciou-se também que as limitações referentes ao tempo de inserção dos profissionais apresentaram-se de forma mais significativa para os profissionais de saúde locados nas USF, sendo mais expressivo no município $\mathrm{B}$. O maior tempo de fixação dos médicos nas USC no município A parece estar associado à forma de contratação dos mesmos. No município B, apesar de se identificar a redução da carga horária dos profissionais das USF, esta não se apresentou como um fator que favoreceu a fixação dos mesmos junto às equipes. Ressalta-se que questões referentes à forma de contratação, ganhos salariais e cumprimento das leis trabalhistas, associadas à estrutura precária das unidades e ausência de incentivos à qualificação profissional também podem estar relacionados a uma maior ou menor fixação dos profissionais de saúde nos municípios.

Uma avaliação sobre o desempenho do PSF no Sul e Nordeste do Brasil revelou que a precarização das formas de contratação de pessoal foi significativamente maior nas USF (51\%) do que nas USC (32\%) e os trabalhadores de saúde informaram baixa satisfação com a estrutura física nos dois modelos de atenção. 19

Estudo realizado por Gil22 apontou como um dos principais nós críticos da ESF a precariedade do vínculo de trabalho, atribuindo-lhe as causas da alta rotatividade das equipes e, consequentemente, o comprometimento do arcabouço da estratégia, principalmente a formação de vínculos e de laços de compromisso e co-responsabilidade entre profissionais de saúde e população. O vínculo com os usuários do serviço de saúde amplia a eficácia das ações de saúde e favorece a participação do usuário durante a prestação do serviço. 23 
O investimento nas políticas de pessoal, voltadas às necessidades dos profissionais que atuam nas equipes é de fundamental importância, principalmente quando se considera que a ESF está efetivamente incorporada à atenção primária como eixo norteador da organização dos sistemas municipais de saúde e vem apresentando uma expansão contínua. Os aspectos relativos à inserção dos profissionais podem apresentar importantes repercussões sobre a continuidade do cuidado aos usuários e sobre o desenvolvimento de novas práticas profissionais que contribuam para a reversão do modelo de atenção à saúde. 23

Embora sejam complexas as redes causais que ligam a estrutura dos serviços de saúde aos seus efeitos e envolvam diversos fatores, tais como as relações interpessoais entre profissionais e gestores do sistema e a organização do processo de trabalho, 24 esses achados reiteram a necessidade de valorização dos componentes de estrutura na avaliação e gestão dos serviços de saúde na sua relação com a qualidade dos processo de trabalho em saúde e para o alcance dos resultados no estado de saúde dos indivíduos e da população.

\section{Referências}

1. Starfield B. Atenção primária: equilíbrio entre necessidades de saúde, serviços e tecnologia. 2 ed. Brasília: UNESCO; 2004. $726 \mathrm{p}$

2. Paim JS. Desafios para a saúde coletiva no século XXI Bahia: Edufba; 2006

3. Teixeira CF. Transição epidemiológica, modelo de atenção à saúde e previdência social no Brasil: problematizando tendências e opções políticas. Ciên Saúde Coletiva. 2004; 9: 841-50.

4. Brasil. Ministério da Saúde. Projeto de Expansão e Consolidação da Estratégia Saúde da Família - Proesf; 2003

5. Brasil. Ministério da Saúde Secretaria de Atenção à Saúde. Departamento de Atenção Primária. Avaliação para melhoria da qualidade da estratégia saúde da família. (Série B. Textos Básicos de Saúde) 6 v. Brasília, DF; 2005.

6. Hartz ZMA, Felisberto E, Viera da Silva LM. Meta-avaliação da atenção básica à saúde - teoria e prática. Rio de Janeiro: Fiocruz; 2008. 410 p.

7. Brasil. Ministério da Saúde, Secretaria de Atenção à Saúde. Departamento de Atenção Básica. Política Nacional de Atenção Básica.. Brasília, DF; 2006.

8. Brasil. Agência Nacional de Vigilância Sanitária. Resolução - RDC (Resolução de Diretoria Colegiada) n. 50: $21 \backslash 02 \backslash 2002$.

9. Brasil. Ministério da Saúde. Secretaria de Atenção à Saúde. Departamento de Atenção Básica. Manual de estrutura física das unidades básicas de saúde: saúde da família. 2 ed. 52 p. (Série A. Normas e Manuais Técnicos). Brasília, DF; 2008 .

10. Donabedian A. The quality of care: how can it be assessed? JAMA. 1988; 260: 1743-8

11. Donabedian A. The definition of quality and approaches to its assessment. In: Explorations in quality assessment and monitoring. vol I. Ann Arbor: Health Administration Press; 1980.

12. Vuori HA. Qualidade em saúde. Divulg Saúde Debate. 1991; 3: 17-25.
13. Vieira da Silva LM, Formigli VLA. Avaliação em saúde: limites e perspectivas. Cad Saúde Pública. 1994; 10: 80-91.

14. Medina MG, Aquino R, Fonseca AC, Assis LN, Vilasbôas ALQ. Caracterização da atenção primária à saúde em municípios que aderiram ao Programa de Saúde da Família. Relatório de Pesquisa; 2008.

15. Brasil. Ministério da Saúde. Portaria GM nº1101. Brasília, DF; 2001.

16. Bahia. Secretaria da Saúde. Superintendência de Vigilância e Proteção da Saúde. Diretoria de Vigilância e Controle Sanitário. BRASIL. Universidade Federal da Bahia. Instituto de Ciências da Saúde. Manual de Biossegurança. Salvador, BA; 2001. Disponível em: http://www.ccs.saude.gov.br/visa/publicacoes/arquivos/P1_ Introdu $\% \mathrm{C} 3 \% \mathrm{~A} 7 \% \mathrm{C} 3 \% \mathrm{~A} 3$ o.pdf

17. Rocha ECA, Araújo MAD. Condições de trabalho das equipes de saúde bucal no Programa Saúde da Família: o caso do Distrito Sanitário Norte em Natal, RN. Rev Adm Pública. 2009; 43: 481-517.

18. Siqueira FCV, Facchini LA, Silveira DS, Piccini RX, Thumé E, Tomasi E. Barreiras arquitetônicas a idosos e portadores de deficiência física: um estudo epidemiológico da estrutura física das unidades básicas de saúde em sete estados do Brasil. Ciênc Saúde Coletiva. 2009; 14: 39-44.

19. Facchini LA, Piccini RX, Tomasi E, Thumé E, Silveira DS, Siqueira FV. Desempenho do PSF no Sul e no Nordeste do Brasil: avaliação institucional e epidemiológica da Atenção Básica a Saúde. Ciênc Saúde Coletiva. 2006; 11: 669-81.

20. Ximenes NFRG, Sampaio JJC. Processo de ascenção ao cargo e as facilidades e dificuldades no gerenciamento do território na Estratégia Saúde da Família. Rev Bras Enferm. 2008, 61: 36-45.

21. Brasil. Ministério da Saúde. Secretaria de Atenção à Saúde, Departamento de Atenção Básica. Avaliação Normativa do Programa Saúde da Família no Brasil: Monitoramento da Implantação e Funcionamento das Equipes de Saúde da Família - 2001/2002. Brasília, DF; 2004. 140 p. Disponível em: http://dtr2001.saude.gov.br/editora/produtos/livros/ pdf/04_0053_M1.pdf 
22. Gil CRR. Formação de recursos humanos em saúde da família: paradoxos e perspectivas. Cad Saúde Pública. 2005; $21: 490-8$

23. Schimith MD, Lima MADS. Acolhimento e vínculo em uma equipe do Programa Saúde da Família. Cad Saúde Pública. 2004; 20: 1487-94.

Recebido em 5 de outubro de 2009

Versão final apresntada em 28 de maio de 2010

Aprovado em 10 de junho de 2010
24. Silveira DS, Santos IS, Costa JSD. Atenção pré-natal na rede básica: uma avaliação da estrutura e do processo. Cad Saúde Pública. 2001; 17: 131-9. 